\title{
Retardo del crecimiento intrauterino asociado con el consumo de pasta básica de cocaína por mujeres gestantes de Bogotá, D.C., Colombia
}

\author{
Oscar Ramírez ${ }^{1}$, Víctor Cárdenas ${ }^{2}$, Germán Peña ${ }^{1}$, \\ Lizette Galindo-Toro ${ }^{1}$, Juan Manuel Lozano ${ }^{3}$ \\ 1 Subdirección de Epidemiologia y Red Nacional de Laboratorios, Instituto Nacional de Salud, Bogotá, D.C., \\ Colombia. \\ 2 Servicio de Epidemiología Aplicada, Subdirección de Epidemiología y Red Nacional de Laboratorios, \\ Instituto Nacional de Salud, Bogotá, D.C.; División de Salud Internacional, Oficina del Programa de \\ Epidemiología, Centers for Disease Control and Prevention, Atlanta, Georgia, EE.UU. \\ ${ }^{3}$ Departamento de Pediatría, Pontificia Universidad Javeriana, Bogotá, D.C., Colombia.
}

El objetivo de este estudio fue identificar una posible asociación entre el consumo de pasta básica de cocaína o 'basuco' entre mujeres embarazadas y el crecimiento intrauterino de sus productos vivos en dos hospitales de tercer nivel en Bogotá, Colombia. Con un diseño de cohorte retrospectiva, se encuestaron 1.878 mujeres en el puerperio mediato para indagar, entre otras variables, la historia sobre el consumo de drogas ilícitas. Tanto a las madres como a sus recién nacidos se les realizaron mediciones antropométricas estandarizadas. Se comparó la prevalencia de bajo peso al nacer (BPN), parto prematuro (PP) y retardo del crecimiento intrauterino (RCIU) con la historia de consumo de drogas ilícitas durante el embarazo por medio de modelos de regresión lineal múltiple y regresión logística múltiple, para ajustar el efecto de posibles confusores como edad materna, estado nutricional y patologias maternas, nivel socioeconómico, escolaridad y tabaquismo, entre otros. Los resultados mostraron que una de cada 189 mujeres consumió sustancias ilegales durante el embarazo, comúnmente Cannabis sativa o pasta básica de cocaína. Las consumidoras de estos productos típicamente tenían menor escolaridad, eran de nivel socioeconómico más bajo, eran más delgadas, con una historia de control prenatal inadecuado, primigestantes o multigestantes y, con frecuencia, también fumaban tabaco y bebian alcohol. Se halló una asociación entre el consumo de las sustancias ilegales con RCIU (cociente de suertes de prevalencias de 5,24, IC95\%: 1,3-21,0), y un déficit de 0,7 desvíos estándares del peso para la edad gestacional $(p=0,03)$. Además, se encontró un cociente de suertes ajustado de 7,78 con IC95\%: 1,0-62, entre el consumo de pasta básica de cocaína y RCIU. Aunque es probable que a través de entrevistas se subestime la prevalencia de consumo de drogas y a pesar del limitado poder del estudio, éste fue capaz de identificar un efecto importante del consumo de pasta básica de cocaína en el RCIU. Estos hallazgos tienen importancia para la salud pública dada la magnitud de proporciones epidémicas que ha cobrado el uso de esta forma de cocaína en las Américas.

Palabras clave: Cannabis, cocaína, retardo del crecimiento intrauterino-etiología, narcóticos, exposición prenatal, tabaquismo-efectos adversos.

Association between the use of free base cocaine (basuco) by pregnant women and intrauterine growth retardation of newborns in Bogota, D.C., Colombia

A retrospective cohort study of 1,878 puerperal women was carried out to assess a possible relationship between the use of free base cocaine or "basuco" by pregnant women and the the risk of intrauterine growth retardation (IUGR) of their live newborns in two hospitals in Bogota, Colombia. We investigated, among other variables, use of illegal drugs using a standardized questionnaire and anthropometric measurements of both the mothers and their newborns. The 
prevalence of low birthweight (LBW), preterm birth (PT), and IUGR was compared to the history of illegal drug consumption during pregnancy, using lineal and logistic multiple regression models in order to adjust for the effect of potential confounders such as maternal age, parity, nutritional status, maternal pathologies, prenatal care, socioeconomic status, educational level, tobacco use, and alcohol consumption. One out of every 189 pregnant women used illegal drugs during their pregnancy, most frequently Cannabis sativa or free-base cocaine. The users of these products were more likely less educated, poorer, did not have prenatal care, and they were tobacco smokers and alcohol drinkers. The study found an adjusted prevalence odds ratio (OR) of 5.24 (IC95\%: 1.3-21.0) for the use of any illegal drug and IUGR, and a deficit of 0.7 standard deviations of weight for their gestational age $(p=0.03)$ among newborns of these drug users. We found an even higher prevalence of IUGR for those newborns of mothers who exclusively smoked free base cocaine $(\mathrm{OR}=7.78, \mathrm{IC} 95 \%: 1.0-62)$. Although it is likely that our method underestimated the true prevalence of drug use, and in spite of its limited statistical power, this study was able to identify an important effect of the use of free base cocaine and the risk of IUGR. These findings bear public health relevance given the fact that the use of this form of cocaine has epidemic proportions in the Americas.

Key words: Cannabis, cocaine, fetal growth retardation-etiology, narcotics, prenatal exposure, smoking-adverse effects.

Se ha informado que el consumo de hidrocloruro de cocaína y cocaína crack es responsable de una serie de resultados adversos del embarazo, entre ellos el retardo del crecimiento intrauterino, aunque también hay resultados contradictorios al respecto (1-8). Sin embargo, es muy escasa la información existente sobre la magnitud y tendencias de la exposición a estas sustancias in utero, particularmente en países productores de estas drogas $(9,10)$. En países de Latinoamérica como Colombia, Venezuela, Bolivia, Perú, Ecuador y Brasil, una parte del consumo de cocaína, principalmente entre personas de escasos ingresos económicos, consiste en fumar directamente, o en mezcla con Cannabis sativa o tabaco, el producto intermedio de la producción de hidrocloruro de cocaína, que se conoce como pasta básica de cocaína, 'basuco', 'susuco' o simplemente 'básica' $(9,10)$. Con el objetivo de describir un posible efecto de la asociación entre el consumo de estas sustancias en mujeres embarazadas y el crecimiento intrauterino de sus recién nacidos, analizamos los datos de un estudio efectuado en dos hospitales de tercer nivel en Bogotá.

Correspondencia:

Victor M. Cárdenas, Texas University, EI Paso. 1100 N. Stanton Ave. 110. El Paso Tejas, 79912.

Recibido: 24/04/00; aceptado: 01/09/00

\section{Materiales y métodos}

\section{Población y diseño del estudio}

Durante el período comprendido entre noviembre de 1994 y marzo de 1995 se realizó un estudio de cohorte retrospectiva entre 2.067 binomios madrenacidos vivos en las primeras 48 horas del puerperio en dos hospitales de tercer nivel en Bogotá para estimar el efecto de ciertos hábitos maternos en los recién nacidos, incluidos, entre otros, el tabaquismo, el consumo de alcohol y el de sustancias ilícitas o de la 'calle'.

Ambos hospitales son de enseñanza y mientras uno de ellos atiende principalmente a la población sin seguridad social, de bajos ingresos y tenía en ese momento la mayor cobertura de atención obstétrica en la ciudad, el segundo atiende a población de clase media.

\section{Criterios de selección}

De los 2.067 binomios madre-hijo elegibles, 189 $(9,1 \%)$ fueron excluidos por ias siguientes causas: haber tenido embarazos múltiples $(n=47)$, recién nacidos con malformaciones mayores $(n=27)$, presentar antes o durante el embarazo enfermedades metabólicas, hormonales o autoinmunes ( $n=66$ ), observaciones faltantes en las principales variables, incluida la edad gestacional $(n=19)$, rechazo $(n=4)$, fallas en el seguimiento de las pacientes en el hospital $(n=21)$ y por causas desconocidas $(n=5)$. 


\section{Recolección de los datos}

Las madres fueron entrevistadas en el puerperio mediato por personal entrenado para tal efecto, siguiendo un cuestionario previamente estandarizado y sin conocimiento del objetivo final del estudio. Se obtuvo información adicional de la historia clínica de la madre. Toda la información del recién nacido se obtuvo de la historia clínica neonatal, consignada directamente por tres de los autores. Los entrevistadores no tuvieron acceso a la información antropométrica de los recién nacidos. Se tomaron mediciones siguiendo un protocolo estandarizado del peso, la talla, el perímetro cefálico y el perímetro torácico del recién nacido. Se tomó el peso en gramos del producto inmediatamente después de nacido, utilizando una báscula electrónica con un margen de error de un decagramo. También se realizaron mediciones de circunferencia braquial y distancia talón-rodilla de las madres.

\section{Variables principales y selección de covariadas}

Variables dependientes. Tanto el peso al nacer, la edad gestacional y el peso para la edad gestacional se analizaron como variables continuas, así como variables dicotómicas. Para hacer el ajuste del peso por la edad gestacional se calcularon puntajes Z, tomando como población de referencia la informada por Wilcox y Skjoerven (11). Las variables dependientes también fueron dicotomizadas usando las siguientes definiciones: bajo peso al nacer $(B P N<2.500 \mathrm{~g})$, parto pretérmino ( $\mathrm{PP}<37$ semanas) y bajo peso para la edad gestacional (RCIU puntaje-Z-2 desviaciones estándar) (12-16). La mayoría de las maternas (1.117/1.878 o $59,5 \%)$ informaron una fecha de última menstruación (FUM) confiable. En un 15,3\% (o 287/1.878), la edad gestacional se calculó con base en ecografía. A un total de 474 productos vivos $(25,2 \%)$ se les estimó la edad gestacional por examen físico y específicamente utilizando la escala de Ballard (15).

Variable independiente y covariadas. El consumo informado de sustancias ilícitas, alguna vez en la vida de la persona, y su uso durante el embarazo fue la variable independiente. Esta variable se agrupó en tres categorías diferentes: 1) las consumidoras de sustancias ilícitas durante el embarazo, 2) las consumidoras de sustancias ilícitas alguna vez en la vida pero que refirieron haberlas abandonado antes de ser gestantes, y 3) las que refirieron no haberlas usado nunca. Esta ultima categoría se utilizó como grupo de referencia para hacer las comparaciones y obtener los estimados de efecto.

Además, se incluyeron en los análisis multivariados las siguientes covariadas: 1) la edad materna en años cumplidos; 2) el número total de años cumplidos de estudio; 3 ) el estado civil (se reagrupó en mujeres con y sin compañero estable); 4) el nivel socioeconómico se midió en una escala ordinal con 6 categorías construidas con base en la estratificación de servicios públicos de su residencia, una medida de hacinamiento (personas por habitación) y el tipo de trabajo de la gestante; 5) una medida compuesta del estado nutricional materno se obtuvo combinando el perímetro braquial y la distancia talón-rodilla, tomando la sumatoria de los puntajes $Z$ de cada una de las medidas individuales $(17) ; 6$ ) hipertensión inducida durante el embarazo; 7) hábito de tabaquismo; 8) el género del recién nacido; 9) el consumo de café; 10) paridad, y, finalmente, 11) el hospital que atendió el parto. Algunas de estas covariadas estuvieron asociadas en este estudio con el riesgo de BPN, PP o RCIU.

\section{Validación}

Se realizó inicialmente una fase piloto del cuestionario, durante la cual se ajustó el texto de las preguntas para lograr su completo entendimiento y estandarización en el contexto de las usuarias de los hospitales seleccionados. Tres de los investigadores supervisaron a los encuestadores permanentemente durante la recolección de la información. Las preguntas sobre hábitos tanto lícitos como ilícitos se realizaron siguiendo las recomendaciones publicadas para su estudio.

Un total de 179 entrevistas se repitieron para medir la reproducibilidad: el estadístico kappa global (k) (18) fue de 0,795, el k interobservador fue de 0,66 y el $k$ intraobservador fue de 0,85 . Se evaluó el potencial del sesgo de memoria y se realizó la entrevista a un subgrupo de 75 gestantes que consultaron al control prenatal en alguno de los 
dos hospitales y, luego, se les repitió la encuesta en el puerperio para evaluar si las madres referían historias de exposiciones diferentes de acuerdo con el peso de los recién nacidos. Al comparar la concordancia no debida al azar entre los recordatorios realizados en la consulta prenatal y los realizados a las mismas gestantes durante el puerperio con la concordancia no debida al azar global durante el puerperio, se encontró que no había diferencias estadísticamente significativas para el consumo de sustancias psicoactivas en general, incluido el uso de basuco, ( $k=0,793$ vs $0,795)$ y otras covariadas, tales como el uso de alcohol $(0,53$ vs 0,72$)$, consulta prenatal $(k=0,88$ vs 0,90$)$ gravidez ( $k=0,91$ vs 0,87$)$ abortos previos ( $k=0,85$ vs 0,89 ), estado civil $(k=0,90$ vs 0,87$)$ y otras exposiciones comunes.

A este mismo grupo de gestantes se le tomó una muestra de sangre y otra de orina, previo consentimiento informado, para medición de cotinina sérica y urinaria como marcador bioquímico de exposición a tabaquismo y como parte de la validación del instrumento de medición. No se midieron metabolitos de otras sustancias psicoactivas. Sin embargo, por problemas técnicos, los resultados finales de estos análisis no fueron confiables, por lo cual no se presentan en este informe.

\section{Análisis estadístico}

Se utilizó un análisis de varianza para las variables continuas(19). Para el análisis de las variables dependientes en escala continua, se realizaron comparaciones de varianza utilizando pruebas de $\mathrm{t}$, así como modelos de regresión lineal múltiple. Se realizó un análisis con cuadros de contingencia para la estimación de las razones de riesgo. Para la modelación multivariada se construyeron variables indicadoras (19). Así mismo, se examinaron los residuales y los cuadros de covarianza de las covariadas para evaluar la idoneidad de la modelación lineal. Para los modelos de regresión logística, se realizó una prueba de Hosmer y Lemeshow (19).

\section{Resultados}

El riesgo de BPN, PP y RCIU fue de $16,2 \%$ (IC95\%: 14,6-18,0), 14,0\% (IC95\%: 12,5-15,7) y
11,1\% (IC95\%: 9,7-12,6), respectivamente. En total, 36 puérperas $(1,9 \%$, IC $95 \%: 1,4-2,7)$ informaron haber usado sustancias ilegales en su vida, de las cuales solamente diez informaron que continuaron el uso de sustancias durante su embarazo. La mitad de estas mujeres informó consumir basuco o cocaína. Cuatro mujeres admitieron haber fumado solamente $C$. sativa. Una materna informó del hábito de inhalar mezclas comerciales de disolventes(20) (toluol, acetonas y xylol), utilizadas como pegamento y conocidas con el nombre local de boxer. Las mujeres que continuaron el uso de alguna de estas sustancias durante el embarazo informaron el consumo diario con mayor frecuencia $(62,5 \%)$ que las mujeres que lo abandonaron durante el embarazo $(15,4 \%)$ $(p=0,017$, prueba de Fisher de dos colas). Aunque sin alcanzar significancia estadística, las mujeres que continuaron el uso de drogas durante el embarazo fumaban pasta básica de cocaína en alguna forma o en combinación con $C$. sativa más frecuentemente $(20 \%)$ que las que no continuaron $(7,1 \%)$ (cuadros 1 y 2 ).

Las consumidoras de sustancias ilegales durante el embarazo difirieron significativamente de las mujeres que informaron no haberlas usado nunca en: 1) tener un promedio menor de escolaridad (5,2 vs 7,9 años); 2 ) ser de nivel socioeconómico bajo (50 vs $16,6 \%$ ); 3 ) ser primigestantes (50 vs $38,4 \%$ ) o tener 4 o más embarazos previos (40 vs $9,8 \%) ; 4$ ) ser más delgadas y de estatura baja (1,46 vs $-0,031$ de puntaje $Z$ ); 5) un control prenatal inadecuado ( 80 vs $34,9 \%$ ); 6 ) fumar cigarrillos de tabaco (90 vs $10,9 \%$ ), y 7) tomar bebidas alcohólicas diaria o semanalmente en mayor porcentaje (10 vs 2,2\%) (cuadro 3 ).

En el cuadro 4 se muestran los estimados ajustados de las variables antropométricas de los recién nacido vivos en escala continua (regresión lineal múltiple), según los grupos de consumidoras ya descritos. Se observa un déficit de $277,4 \mathrm{~g}$ de peso en el grupo de consumidoras de sustancias ilegales durante el embarazo con respecto al grupo de referencia (prueba de $t$, valor de $p=0,12$ ). Así mismo, se encontró una diferencia en el puntaje $Z$ del peso para la edad gestacional de $-0,71$, indicando un déficit aproximado de $70 \%$ de una desviación estándar a lo largo de la escala de 
Cuadro 1. Características del uso entre las consumidoras antes y durante el embarazo

\begin{tabular}{lcccc}
\hline Características & $\begin{array}{c}\text { Consumidoras de } \\
\text { "drogas ilícitas" pero } \\
\text { no durante el embarazo }\end{array}$ & $\begin{array}{c}\text { Consumidoras de } \\
\text { "drogas ilícitas" } \\
\text { durante el embarazo }\end{array}$ \\
\hline Consumo diario (\%) & $4 / 26^{*}$ & 15,4 & $5 / 8^{*}$ & $62,5^{* *}$ \\
Uso de marihuana (\%) & $18 / 26$ & 64,3 & $4 / 10$ & 40 \\
Uso de basuco (\%) & $3 / 26$ & 10,7 & $2 / 10$ & 20 \\
Uso de marihuana y basuco (\%) & $2 / 26$ & 7,1 & $2 / 10$ & 20 \\
Cualquier tipo de policonsumo (\%) & $3 / 26$ & 11,5 & $3 / 10$ & 30 \\
\hline
\end{tabular}

* Dos gestantes sin datos

** Valor de $\mathrm{p}=0,017$

Cuadro 2. Tipo de "droga ilícita" consumida durante el embarazo e informada en la encuesta

\begin{tabular}{lcc}
\hline Tipo de "droga ilícita" & Número & $\%$ \\
\hline Marihuana y basuco & 2 & 20 \\
Marihuana y boxer* & 1 & 10 \\
Solamente marihuana & 4 & 40 \\
Solamente basuco** & 2 & 20 \\
Solamente cocaína*** & 1 & 10 \\
Total & 10 & 100 \\
\hline
\end{tabular}

* Pegante con un compuesto del tolueno y otros solventes

** Sulfato de cocaína

${ }^{* * *}$ Clorhidrato de cocaína

pesos por edad gestacional (prueba de $t$, valor de $p=0,03$ ). No se encontraron diferencias cuando se compararon los promedios y varianzas de la edad gestacional. Del mismo modo, no se encontraron diferencias significativas entre el grupo de mujeres que no utilizaron sustancias ilegales durante el embarazo y el grupo de referencia. No hubo diferencias estadísticamente significativas en el índice ponderal $(12,13,16)$, ni en la relación perímetro cefálico/perímetro torácico. Estas medidas se utilizaron para evaluar la simetría en las medidas de los recién nacidos vivos e indicaron que el RCIU fue principalmente simétrico. Sin embargo, se observaron déficits del orden de 1,3 $\mathrm{cm}, 1 \mathrm{~cm}$ y de $1,3 \mathrm{~cm}$ en las tallas, los perímetros cefálicos y los perímetros torácicos entre los productos de no usuarias y las usuarias durante el embarazo. Estas diferencias no fueron estadísticamente significativas. Cuando se compararon los promedios de índices corporales y los promedios de la razón entre el perímetro cefálico y el perímetro torácico del grupo de consumidoras durante el embarazo con el de las mujeres que nunca usaron sustancias, tampoco se encontraron diferencias estadísticamente significativas.

Se encontró una fuerte asociación, principalmente con RCIU (cuadro 5), estimada por una razón de suertes ajustada por las covariadas ya mencionadas de 5,2, con intervalos de confianza que van de 1,3 a 20,0 . El cuadro 5 presenta también medidas de efecto en el riesgo de BPN (CS $=4,9$; IC95\%:1,3-19,0) y PP (CS=0,6; IC95\%: $0,1-5,1)$.

Restringiendo el análisis al dominio de las mujeres que solamente consumieron pasta básica de cocaína, comparadas de nuevo con las mujeres que informaron no haberlas usado, la regresión logística múltiple indicó que el riesgo de RCIU, BPN y PP fue, respectivamente, de 7,8 (IC95\%: 1,0-62,0), 9,8; IC95\%: 1,0-103,9) y 1,0 (IC95\%: 0,1-10,9).

\section{Discusión}

Antes de entrar a discutir las implicaciones de los hallazgos presentados, consideraremos las siguientes limitaciones de este estudio. Una de ellas estriba en el limitado número de gestantes expuestas durante el embarazo a sustancias ilícitas, por lo que el poder del estudio es apenas razonable para detectar cociente de suertes de 6 con un poder razonable $(80 \%)$ en las tasas observadas de BPN, PP y RCIU (18).

Otras debilidades en la validez de los resultados encontrados, se originan principalmente en la forma de indagación retrospectiva empleada para estimar el consumo de sustancias. Por el hecho mismo de que su consumo es ilegal, es muy probable que la prevalencia de exposición esté subestimada y que quienes admitieron el consumo 


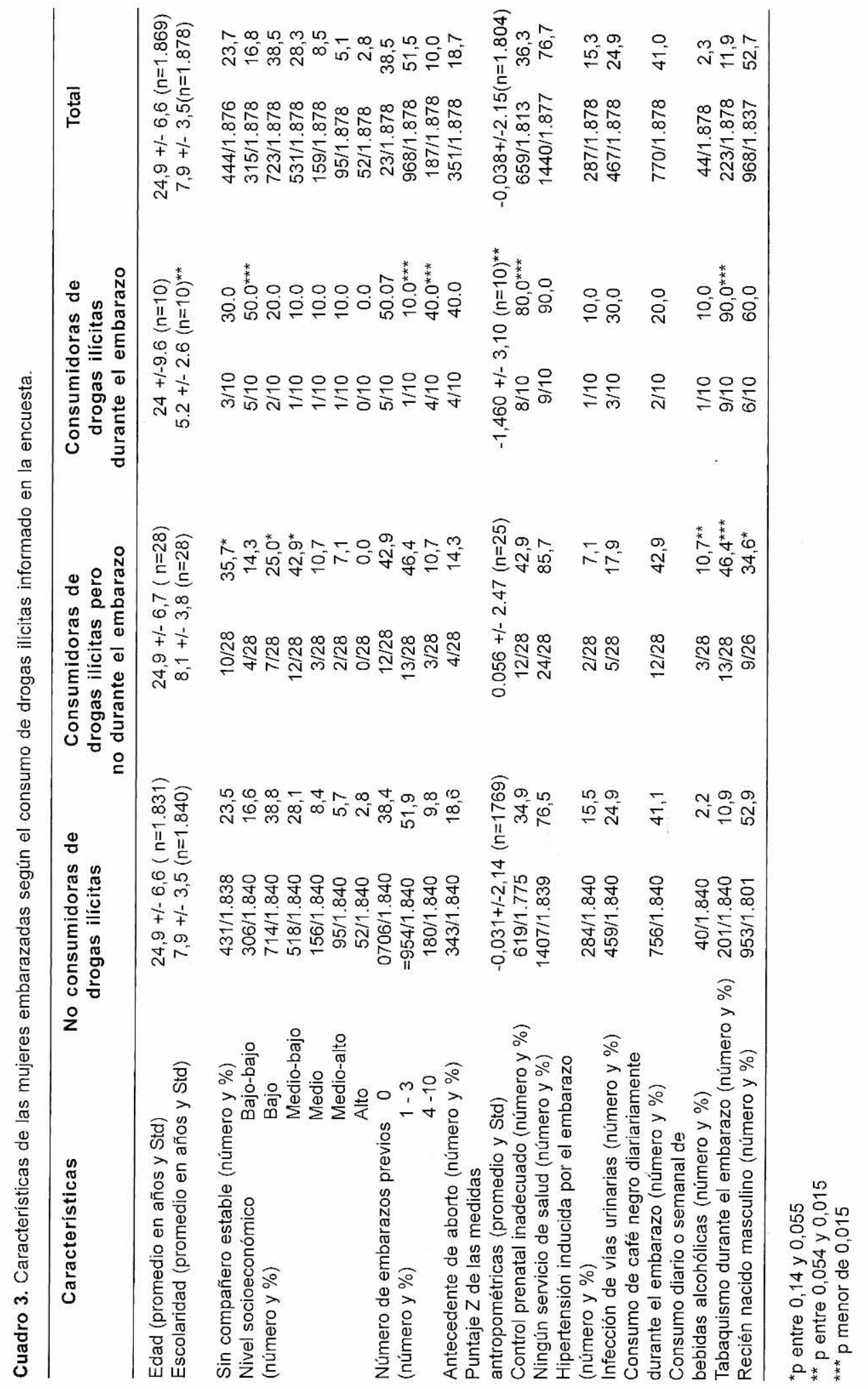


Cuadro 4. Comparación de medias de las variables antropométricas de los recién nacidos de acuerdo con la historia de consumo materno de drogas ilícitas.

\begin{tabular}{lccc}
\hline \multicolumn{1}{c}{$\begin{array}{c}\text { Variables } \\
\text { (Estimados ajustados) }\end{array}$} & $\begin{array}{c}\text { No consumidoras de } \\
\text { drogas ilícitas }\end{array}$ & $\begin{array}{c}\text { Consumidoras de } \\
\text { drogas ilícitas pero } \\
\text { no durante el embarazo }\end{array}$ & $\begin{array}{c}\text { Consumidoras de } \\
\text { drogas ilícitas } \\
\text { durante el embarazo }\end{array}$ \\
\hline Peso (g) & $3.164,6$ & $3,125,9$ & $2.865,8$ \\
Valor estimado, error estándar del coeficiente B & 85,75 & 107,78 & 181,0 \\
y valor de p & & 0,73 & 0,097 \\
Edad gestacional (días) & 272,1 & 268,9 & 271,1 \\
Valor estimado, error estándar del coeficiente B & 2,57 & 3,24 & 5,41 \\
y valor de p & & 0,35 & 0,86 \\
Puntaje Z del peso según edad gestacional & $-0,349$ & $-0,253$ & $-1,069$ \\
Valor estimado, error estándar del coeficiente B & 0,156 & 0,199 & 0,330 \\
y valor de p & & 0,54 & 0,028 \\
Talla (cm) & 49,9 & 49,4 & 48,4 \\
Valor estimado, error estándar del coeficiente B & 0,44 & 0,56 & 0,93 \\
y valor de p & & 0,45 & 0,12 \\
Indice ponderal & & 24,4 & 24,7 \\
Valor estimado, error estándar del coeficiente B & 25,1 & 0,74 & 1,23 \\
y valor de p & 0,58 & 0,30 & 0,74 \\
Perímetro cefálico (cm) & 33,7 & 33,2 & 32,7 \\
Valor estimado, error estándar del coeficiente B & 0,29 & 0,37 & 0,61 \\
y valor de p & & 0,11 & 0,10 \\
Perímetro torácico (cm) & 32,8 & 33,1 & 31,4 \\
Valor estimado, error estándar del coeficiente B & 0,40 & 0,50 & 0,87 \\
y valor de p & & 0,57 & 0,12 \\
Razón entre el perímetro cefálico y el & 0,962 & 0,950 & 0,928 \\
perímetro torácico & & & 0,066 \\
Valor estimado, error estándar & & 0,039 & 0,61 \\
de coeficiente B y valor de p & 0,031 & 0,75 & \\
\hline
\end{tabular}

*Todos los valores fueron ajustados por las siguientes variables en regresión lineal múltiple: edad, sin compañero estable, nivel socioeconómico, embarazos previos, antropometría materna, hipertensión inducida por el embarazo, infección de vias urinarias, consumo de café negro diariamente, consumo diario o semanal de bebidas alcohólicas, hábito tabáquico materno y sexo del recién nacido.

** Grupo de comparación

- Indice ponderal=peso/talla ${ }^{3}$

Cuadro 5. Medidas de riesgo para bajo peso al nacer, pretérmino y retardo del crecimiento intrauterino según consumo de drogas ilicitas.

\section{(Estimados ajustados)* \\ No consumidoras de drogas ilícitas

Consumidoras de
drogas ilícitas pero
no durante el embarazo no durante el embarazo}

Consumidoras de

drogas ilícitas durante el embarazo

\begin{tabular}{cccc}
\hline $\begin{array}{c}\text { Bajo peso al nacer }(<2.500 \mathrm{~g}) \\
\text { (OR e IC 95\%) }\end{array}$ & 1 & 1,81 & 4,90 \\
Recién nacidos pretérminos (<37 sem) & 1 & $0,68-4,80$ & $1,30-19,0$ \\
(OR e IC 95\%) & & 2,26 & 0,63 \\
Retardo del crecimiento intrauterino & 1 & $0,85-6,02$ & $0,10-5,10$ \\
(OR e IC 95\%) & & 1,48 & 5,24 \\
\hline
\end{tabular}

*Todos los estimados fueron ajustados por las siguientes variables con regresión logística múltiple: edad, sin compañero estable, nivel socioeconómico, embarazos previos, antropometría materna, hipertensión inducida por el embarazo, infección de vías urinarias, consumo de café negro diariamente, consumo diario o semanal de bebidas alcohólicas, hábito tabáquico materno y sexo del recién nacido

${ }^{* *}$ Grupo de comparación

- Igual o menor de dos desviaciones estándares de la población de referencia (ver texto). 
de tales sustancias se hubiesen expuesto efectivamente. Hay más incertidumbre acerca de la dirección en que podrían haberse sesgado los resultados por recordatorio diferencial sobre el consumo en el grupo de mujeres que tuvieron un desenlace adverso del embarazo (BPN, PP o $\mathrm{RCIU})$ : teóricamente, algunas podrían asignarle un papel causal y quizás informarlo más que quienes tuvieron un producto vivo normal; alternativamente, por la presión social existente, algunas mujeres podrian negar haberlas consumido.

La mayor parte de las edades gestacionales (EG) se estimaron utilizando la FUM confiable o extrapolándola de los resultados de una ecografía temprana. En el grupo en el que no se tenían estos datos fue necesario utilizar la escala de Ballard, cuya concordancia con la edad gestacional es aceptable $(15,16)$; seguramente, el haber utilizado este tipo de medición de la EG introdujo error a la variable RCIU, pero lo más probable es que éste sea del tipo no diferencial. Por el contrario, haber excluido estas maternas seguramente habría producido un sesgo de selección en los estimados, cuya dirección sería difícil de determinar.

En el presente trabajo no se utilizaron marcadores bioquímicos para validar el instrumento de recolección de la información sobre exposición a sustancias psicoactivas. Los marcadores bioquímicos se han utilizado, preferencialmente, en estudios en los que durante un programa establecido de control prenatal se realizan las pruebas rutinariamente durante ciertos momentos del embarazo. Por la vida media de estos metabolitos, su medición ha sido muy discutida durante el parto o en el puerperio, ya que durante este período lo usual es que no haya consumo. Por tanto, habría un aumento en el número de pruebas falsas negativas y una subestimación del consumo. Ello, así como la baja prevalencia de consumidoras que persisten en el uso hasta el final del embarazo y los altos costos de estas pruebas, hace que la medición rutinaria de marcadores bioquímicos en este tipo de estudio se considere poco eficiente.

La submuestra de gestantes que se evaluaron durante alguna visita prenatal era muy pequeña para esperar tener suficientes consumidoras que justificaran el uso de estos marcadores. Sin embargo, otros estudios, notablemente los que han utilizado marcadores bioquímicos del consumo de sustancias, han hallado una asociación como la aquí informada $(1,3,5,21-23)$.

Aunque el diseño del estudio presenta algunas limitantes como las comentadas anteriormente, también permitió obtener información sobre un grupo de maternas que, de otra forma, se hubiera perdido. Si el diseño de este estudio hubiera sido de cohorte prospectiva basado en las gestantes que asistian a control prenatal, los resultados tendrían un sesgo de selección importante, ya que según nuestros hallazgos, $80 \%$ de las consumidoras durante el embarazo no tuvieron un control prenatal adecuado.

La prevalencia de bajo peso al nacer encontrada en este estudio fue un poco más elevada de la informada en otros estudios realizados en la ciudad, en los cuales la prevalencia está alrededor de $10 \%$ (24). Esto es explicable en parte por el tipo de población seleccionada, más bien representativa de embarazos de mediano a alto riesgo. Nuestros datos sugieren, así mismo, que entre estas consumidoras de drogas de la calle existe mayor tendencia al policonsumo, principalmente cualquier forma de cocaína, sin haberlo abandonado durante el embarazo. Esto nos hace pensar que las conclusiones sobre la asociación encontrada se deben limitar al consumo 'pesado' de drogas psicoactivas y, entre ellas, a la pasta básica de cocaína:

Es notable que de acuerdo con nuestras observaciones y las de otros autores $(9,10)$, la prevalencia de consumo de sustancias ilegales en Colombia es menor que la informada en otros países, en algunos de los cuales la prevalencia de consumo en gestantes ha sido alrededor de $15 \%(1-6,25)$.

El abuso y la dependencia de sustancias psicoactivas puede iniciarse y perpetuarse debido a un amplio rango de factores fisiológicos, del comportamiento y socioeconómicos (26). Como se ha descrito en diferentes estudios en otros países, la población consumidora de drogas ilícitas durante el embarazo corresponde a una minoría de gestantes muy bien definidas, en las 
cuales las características principales son la pobreza, la baja escolaridad, la malnutrición y la multiparidad (21,27-29).

Estos hallazgos también concuerdan con una encuesta realizada en Colombia en 1999 en una muestra que incluyó 307.713 jóvenes en 29 ciudades capitales y 21 no capitales, en el que se describe que las características de los consumidores de sustancias psicoactivas son la baja escolaridad, el desempleo y el tener entre 20 y 24 años de edad (30).

Hay evidencias de que muchos de los abusadores de este tipo de drogas tienen trastornos psicológicos y psicosociales que contribuyen a la dependencia y que a su vez se mezclan y se agravan con ésta $(27,28)$.

Las gestantes que dijeron haber dejado el hábito antes de quedar en embarazo, presentaban mayor escolaridad y un nivel socioeconómico más alto que las mujeres que continuaron el consumo durante el embarazo. Aparentemente, quienes abandonaron los hábitos podrían representar al grupo de consumidoras ocasionales o 'ligeras', con mayor tendencia al uso de sustancias adictivas como el cigarrillo y el alcohol, con incursión limitada en el consumo de sustancias ilícitas. En este grupo no se encontró efecto sobre el crecimiento fetal, aunque sí se observa un mayor riesgo de nacidos vivos pretérminos, que no alcanzó significancia estadística.

Se han descrito diferentes efectos tóxicos producidos por drogas de abuso, especialmente por cocaína, que incluyen anormalidades congénitas, fundamentalmente en el tracto genitourinario, un aumento en la incidencia de enfermedad cerebrovascular fetal, efectos en la ontogenia del sistema nervioso central (31-33), trastornos del crecimiento fetal y efectos sobre el comportamiento fetal, además de abortos espontáneos, muerte fetal, placenta previa y abrupcio de placenta (1,3,31-42). Recientemente se ha informado del efecto con gradiente biológico por el cual recién nacidos de 3 semanas de edad exhibían una mayor excitabilidad a mayor dosis de cocaína in utero (43).

Se postula que el efecto de la cocaína sobre el crecimiento fetal se debe principalmente a trastornos de la oxigenación fetal, ya que reduce el flujo uterino y altera el intercambio transplacentario de oxigeno al feto. Los principales efectos descritos en modelos animales son los de un aumento en la resistencia vascular uterina y en la presión arterial sistémica materna, así como disminución del flujo uterino. Estas respuestas se acompañaron de hipoxemia fetal marcada, hipertensión y taquicardia (44). Tampoco observamos una mayor incidencia de hipertensión inducida por el embarazo y abrupcio de placenta (45-47) (resultados no presentados).

Estos hallazgos son consistentes con otras observaciones sobre el efecto del consumo de drogas ilícitas en gestantes y resultados adversos del embarazo como BPN, PP y RCIU $(1,3,21,34-$ $37,39,40)$, especialmente con clorhidrato de cocaína y crack.

Este informe provee evidencia adicional de que una forma importante del consumo de cocaína epidémico en Suramérica desde finales de los 70 , o sea el de la pasta básica de cocaína, tiene efectos deletéreos en la salud semejantes a los informados para el clorhidrato de cocaína y el crack, aunque diferentes en su forma, ya que el clorhidrato de cocaína es un polvo que se inhala o inyecta y el crack es una forma fumable de cocaína a la que le han removido algunas de las impurezas presentes en la pasta básica. Entre las sustancias presentes en la pasta básica se ha informado queroseno, plomo, ácido sulfúrico, metanol, ácido benzoico, éter, talcos y otros compuestos del silicio.

En Colombia, se han realizado varios estudios sobre la prevalencia del uso de sustancias $(9,10,30)$. Una encuesta conducida en 1991 incluyó una muestra probabilística de hogares, con entrevistas a 28.698 personas mayores de 11 años (10). De acuerdo con esta encuesta, 5,9\% de los entrevistados informaron haber consumido alguna droga ilegal en su vida. Uno y medio por ciento había consumido cocaína o pasta básica de cocaína. La encuesta también indagó sobre el consumo de estas sustancias durante el embarazo: $85 \%$ de las mujeres que usualmente consumen sustancias ilegales las abandonan durante el embarazo. 
En otro estudio más reciente ya mencionado (30), el uso, alguna vez en la vida, de marihuana, cocaína o pasta básica de cocaína informado por las mujeres fue de $5,8,1,9$ y $1,1 \%$, respectivamente, y el uso de las mismas sustancias en el último mes previo a la encuesta fue de 2,1, 0,6 y $0,5 \%$, respectivamente.

Nuestro estudio estima que la prevalencia de uso es de 5/1.000 embarazadas y una prevalencia de 20,2/1.000 de uso de por vida en mujeres en edad fértil en Colombia.

Si se toman las tasas de consumo en el último mes como las más representativas de las consumidoras habituales y se considera que alrededor de $85 \%$ abandona el consumo durante el embarazo, entonces se podría estimar en 4,5/ 1.000 la prevalencia del uso habitual de sustancias psicoactivas durante el embarazo.

No obstante, las tasas encontradas en nuestro estudio son mayores y ello bien podría deberse a que los hospitales en que se realizó el estudio son de referencia para embarazos y partos de mayor riesgo.

Nuestros hallazgos señalan un área de investigación y de acción para la salud pública en Latinoamérica que ha sido poco reconocido y que amerita ser considerado por los programas de salud reproductiva.

\section{Agradecimientos}

A los directivas del Instituto Nacional de Salud, Instituto Materno Infantil, Hospital San Ignacio y Universidad Javeriana , que apoyaron la realización del estudio.

\section{Referencias}

1. Robins LN, Mills JL. Effects of in utero exposure to street drugs. Am J Public Health 1993;83(Suppl.).

2. Kramer MS. Determinants of low birth weight: methodological assessment and meta-analysis. Bull WHO 1987;65:663-737.

3. Eyler FD, Behnke M, Conlon M, Woods NS, Wobie K. Birth outcome from a prospective, matched study of prenatal crack/cocaine use. I. Interactive and dose effects on health and growth. Pediatrics 1998;101:229-37.

4. Plessinger MA, Woods JR Jr. Cocaine in pregnancy. Recent data on maternal and fetal risks. Obstet Gynecol Clin North Am 1998;25:99-118.
5. Wagner CL, Katikaneni LD, Cox TH, Ryan RM. The impact of prenatal drug exposure on the neonate. Obstet Gynecol Clin North Am 1998;25:169-94.

6. Hanna-EZ, Faden-VB, Dufour-MC. The effects of substance use during gestation on birth outcome, infant and maternal health. J Subst Abuse 1997;9:111-25.

7. Hulse GK, English DR, Milne E, Holman CD, Bower Cl. Maternal cocaine use and low birth weight newborns: a meta-analysis. Addiction 1997;92:1561-70.

8. Richarson GA, Hamel SC, Goldschmidt L, Day NL. Growth of infants prenatally exposed to cocaine/crack: comparision of prenatal care and a no prenatal care sample. Pediatrics 1999;104:1-10.

9. Posada JA, Torres Y, Calderón H. Estudio nacional de salud mental y consumo de sustancias psicoactivas, Colombia 1993. Santa Fe de Bogotá: Ministerio de Salud; 1995.

10. Torres Y, Estupiñan DE. Sustancias psicoactivas en Santafé de Bogotá. Encuesta Nacional sobre Conocimientos, actitudes y prácticas en salud. Bogotá: Instituto Nacional de Salud; 1991.

11. Wilcox AJ, Skjoerven R. Birth weight and perinatal mortality: the effect of gestational age. Am J Public Health 1992;82:378-82.

12. Manning F. Fetal medicine principles and practice. New York: Appleton and Lange; 1995.

13. Patterson RM. Definition, epidemiology, and morphometric classification of intrauterine growth retardation. En: Divon MY, editor. Abnormal fetal growth. Amsterdam: Edit. Elsevier; 1991.

14. Kiely JL, Susser M. Preterm birth, intrauterine growth retardation and perinatal mortality. Am J Public Health 1992;82:343-5.

15. Ballard JL, Novak KK, Driver M. A simplified score for assesment of fetal maturation of newly born infants. J Pediatr 1979.95:769-74.

16. Sparks JW, Cetin Y. Intrauterine growth and nutrition. Fetal and neonatal physiology. Philadelphia: W.B. Saunders; 1992

17. WHO. Maternal anthropometry and pregnancy outcomes. A WHO collaborative study. Bulletin WHO 1995; 73(Suppl.).

18. Fleiss JL. Statistical methods for rates and proportions. Second edition. New York; John Wiley \& Sons; 1981.

19. Kleinbaum DG, Kupper LL, Muller KE. Applied regression analysis and other multivariable methods. Second edition. Boston: PWS-Kent; 1988.

20. Jones HE, Balster RL. Inhalant abuse in pregnancy. Obstet Gynecol Clin North Am 1998;25:153-67.

21. Handler A, Kistin N, Davis F, Ferré C. Cocaine use during pregnancy: perinatal outcomes. Am J Epidemiol 1991;133:818-25. 
22. Neuspiel DR, Markowitz M, Drucker E. Intrauterine cocaine, lead, and nicotine exposure and fetal growth. Am J Public Health 1994;84:1492-5.

23. Ostrea EM Jr., Ostrea AR, Simpson PM. Mortality within the first 2 years in infants exposed to cocaine, opiate, or cannabinoid during gestation. Pediatrics 1997;00:79-83.

24. Ministerio de Salud-UNICEF. Peso al nacer en cinco ciudades colombianas. Bogotá: Ministerio de Salud; 1991.

25. AAP, Committee on Substance Abuse. Tobacco, Alcohol, and Other Drugs. The role of the pediatrician in prevention and management of substance abuse. Pediatrics 1998;83:125-8.

26. Mendelson JH, Mello NK. Management of cocaine abuse and dependence. N Eng J Med 1996;334:96372 .

27. Chazotte C, Youchah J, Freda MC. Cocaine use during pregnancy: the impact of prenatal care and drug treatment. Semin Perinatol 1995;19:293-300.

28. Amaro H, Zuckerman B, Cabral H. Drug use among adolescent mothers: profile of risk. Pediatrics 1989; $84: 144-51$

29. Knight EM, James H, Edwards $\mathrm{CH}$, Spurlock BG. Oyemade UJ, Johnson AA, et al. Relationships of serum illicit drug concentrations during pregnancy to maternal nutritional status. J Nutr 1994;124:973S-80S.

30. Presidencia de la República. Plan Nacional de Lucha contra las Drogas. Colombia 1998-2002. Bogotá, D.C.: Programa Rumbos; 1999.

31. Mayes LC. Developing brain and in utero cocaine exposure: effects on neuroontogeny. Dev Psychopathol 1999;11:685-714.

32. Ferriero DM, Dempsey DA. Impact of addictive and harmful substances on fetal brain development. Curr Opin Neurol 1999;12:161-6.

33. Wickelgren I. Cocaine wreaks subtle damage on developing brains. Science 1997;278:38-9.

34. Zuckerman B, Frank BA, Hingson R, Amaro H, Levenson SM, Kayne $\mathrm{H}$, et al. Effects of maternal marijuana and cocaine use on fetal growth. $\mathrm{N}$ Engl J Med $1989 ; 320: 762-8$
35. Zuckerman B, Frank BA, Hingson R, Amaro $\mathbf{H}$, Levenson SM, Kayne $H$, et al. Cocaine use during pregnancy: prevalence and correlates. Pediatrics 1988; $82: 888-95$

36. Hatch EE, Bracken MB. Effect of marijuana use in pregnancy on fetal growth. Am J Epidemiol 1986;124: 986-93.

37. Chouteau M, Brickner N, Leppert P. The effect of cocaine abuse on birth weight and gestational age. Obstet Gynecol 1988;72:351-4.

38. Chasnoff IJ, Burns WJ, Schnoll SH, Burns KA. Cocaine use in pregnancy. N Eng J Med 1985;313:666-9.

39. Wagner CL, Katikaneni LD, Cox TH, Ryan RM. The impact of prenatal drug exposure on the neonate. Obstet Gynecol Clin North Am 1998;25:169-94.

40. Singer L, Arendt R, Song LY, Warshawsky E, Kliegman R. Direct and indirect interactions of cocaine with childbirth outcomes. Arch Pediatrics Adolesc Med 1994;148:959-64

41. Kosofsky BE. Cocaine-induced alterations in neurodevelopment. Semin Speech Lang 1998;19:109-21.

42. Napiorkowski B, Lester BM, Freier MC, Brunner S, Dietz L, Nadra A, et al. Effects of in utero substance exposure on infant neurobehavior. Pediatrics 1996;98:71-5.

43. Tronick EZ, Frank DA, Cabral H, Mirochnick M, Zuckerman B. Late dose-response effects of prenatal cocaine exposure on newborn neurobehavioral performance. Pediatrics 1996;98:76-83.

44. Woods JR Jr., Plessinger MA, Clark KE. Effect of cocaine on uterine blood flow and fetal oxygenation. JAMA 1987:257:957-61.

45. Mooney EE, Boggess KA, Herbert WN, Layfield LJ. Placental pathology in patients using cocaine: an observational study. Obstet Gynecol 1998;9:925-9.

46. Hulse GK, Milne E, English DR, Holman CD. Assessing the relationship between maternal cocaine use and abruptio placentae. Addiction 1997;92:1547-51.

47. Macones GA, Sehdev HM, Parry S, Morgan MA, Berlin JA. The association between maternal cocaine use and placenta previa. Am J Obstet Gynecol 1997;177:1097-100. 